\title{
Crystalline-Vitreous Interface in Two Dimensional Silica
}

\author{
Leonid Lichtenstein, Markus Heyde, ${ }^{*}$ and Hans-Joachim Freund \\ Fritz-Haber-Institut der Max-Planck-Gesellschaft, Faradayweg 4-6, 14195 Berlin, Germany
}

(Received 22 May 2012; published 6 September 2012)

\begin{abstract}
The interface between a crystalline and a vitreous phase of a thin metal supported silica film was studied by low temperature scanning tunneling microscopy. The locally resolved evolution of Si-Si nearest neighbor distances and characteristic angles was evaluated across the border. Furthermore, we investigated the behavior of the ring size distribution close to the crystalline-vitreous transition. The crystalline order was found to decay gradually within about $1.6 \mathrm{~nm}$ into the vitreous state.
\end{abstract}

DOI: 10.1103/PhysRevLett.109.106101

PACS numbers: 68.37.Ef, 68.35.bj, 68.35.Rh, 68.47.Gh

The topological transition from a crystalline to an amorphous material can result in very complex structures. It is a demanding task to investigate the atomic arrangement of such boundaries and it requires a well-defined structural model system. Understanding the interface between a crystal and a glass can lead to a better description of the crystal-to-glass and the liquid-to-glass transitions. In addition, fundamental knowledge about semicrystalline materials can be gained, because there the crystallites are separated by disordered boundary regions.

A vast amount of studies was published concerning crystalline-amorphous $(c-a)$ interfaces of different, mostly tetrahedrally coordinated materials, including $c-\mathrm{Ge} / a-\mathrm{Ge}$ [1], $c$-Si/a-Ge [2,3], $c$-Si/a-Si [1,4-7], $c-\mathrm{Al}_{2} \mathrm{O}_{3} / a-\mathrm{CaSiO}_{3}$ [8], $c-\beta-\mathrm{Si}_{3} \mathrm{~N}_{4} / a-\mathrm{SiO}_{2}$ [9], and $c-\mathrm{Si} / a-\mathrm{SiO}_{2}$ [10-21], the last example being an important interface in semiconductor technology. The transitional structures were investigated by a large variety of theoretical $[1,4,6,8,14,16,17,19]$ and experimental methods $[2,3,5,9-13,15,18,20,21]$. However, the interface between crystalline and vitreous silica has not been addressed so far. Moreover, the application of scanning probe microscopy to a $c$ - $a$ transition has not been shown. These structures were thought to be inaccessible by scanning probe microscopy, because the interfaces are buried inside the bulk materials [3].

In a recent publication, we reported on the atomic structure of a thin vitreous bilayer silica film on a $\mathrm{Ru}(0001)$ support using low temperature scanning tunneling microscopy (STM) [22]. The film's structure exhibited high short range order, but no long range periodicity. Rings with four to nine $\mathrm{Si}$ and $\mathrm{O}$ atoms were observed. The ring size distribution of the vitreous film exhibits a characteristic log-normal behavior, which is due to the connectivity requirements of two dimensional (2D) random networks [23] (see Supplemental Material [24], Figure S5). By comparing the pair correlation functions, we could prove that the 2D film is a good model of a three dimensional (3D) glass. Our results were confirmed by transmission electron microscopy experiments of $2 \mathrm{D}$ vitreous silica prepared on graphene [25]. Furthermore, we showed that the silica film can also be grown in a crystalline phase [26]. We compared the characteristic distances and angles occurring in both the crystalline and the vitreous phase of the thin silica film in great detail, showing good agreement to bulk silica materials [27]. The silica film on $\mathrm{Ru}(0001)$ provides the unique opportunity to study the interface between crystalline and vitreous growth modes with atomic resolution in real space.

Herein, we report on a detailed evaluation of the one dimensional (1D) interface between a crystalline and a vitreous region of the thin silica film. The evolution of characteristic atomic distances and angles is evaluated in detail. We discuss how ring statistics change across such a border. A measure of the film's crystallinity is introduced, and we look at its development from the crystalline to the glassy phase.

In this study, we applied a custom-built dual mode microscope which combines noncontact atomic force microscopy and STM using a tuning fork sensor. The microscope is operated at low temperatures $(5 \mathrm{~K})$ in ultrahigh vacuum. Prior to film preparation, the $\mathrm{Ru}(0001)$ substrate was cleaned by repeated cycles of $\mathrm{Ar}^{+}$bombardment at $1 \mathrm{kV}$ and annealing to $1500 \mathrm{~K}$. The cleanliness of the substrate was controlled by low energy electron diffraction and STM. The silica films were prepared by evaporating $\mathrm{Si}$ from a $\mathrm{Si}$ rod onto a 3O- $(2 \times 2)$-precovered $\mathrm{Ru}(0001)$ surface in an $\mathrm{O}_{2}$ atmosphere of $2 \times 10^{-7}$ mbar. Subsequently, the sample was annealed at $1180 \mathrm{~K}$ in $2 \times$ $10^{-6}$ mbar $\mathrm{O}_{2}$, resulting in an extended and flat silica bilayer. Depending on the initial $\mathrm{Si}$ coverage and the cooling rate, we could grow vitreous silica films or films with coexisting crystalline and vitreous regions [28].

Figure 1(a) shows an atomically resolved STM image of a crystalline-vitreous transition region in the silica film. The porous structure of the bilayer film is clearly visible. Furthermore, every pore exhibits atom-sized protrusions. Because every four bright spots are arranged in a threebladed windmill shape, we assign these features to the positions of $\mathrm{Si}$ atoms. The coordinates of the $\mathrm{O}$ atoms were obtained by calculating the center between every pair of Si-Si nearest neighbors (NNs). The final atomic 

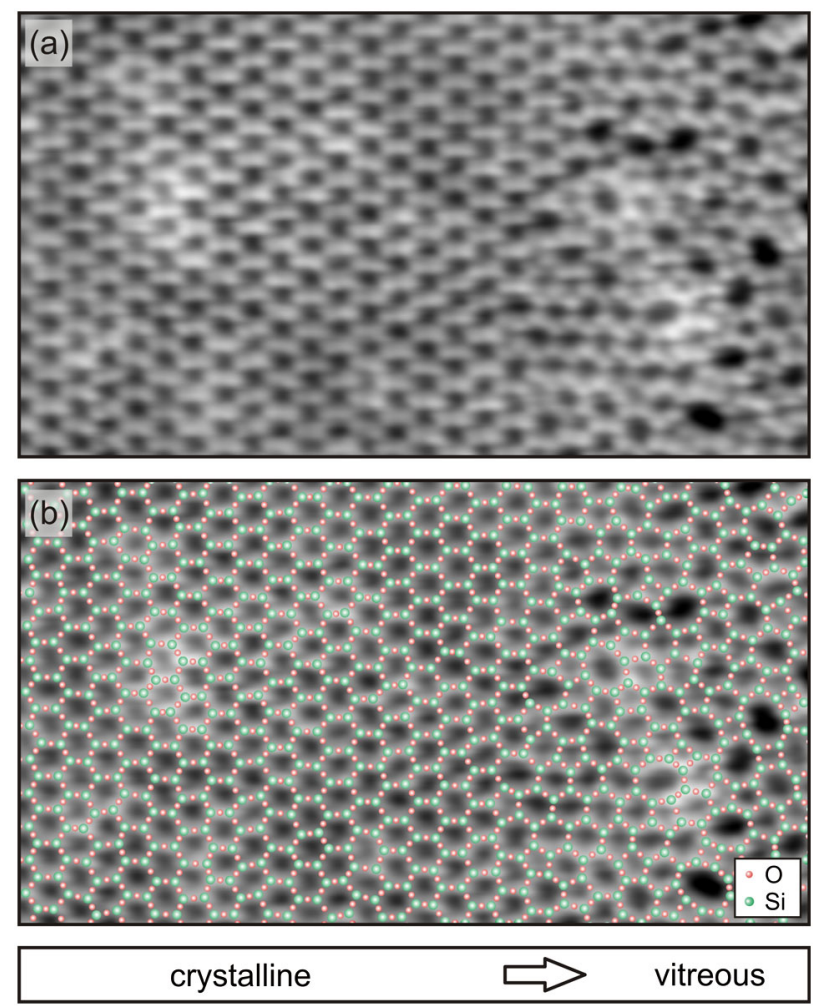

FIG. 1 (color online). (a) Atomically resolved STM image of the crystalline-vitreous interface in the silica film $\left(V_{S}=2 \mathrm{~V}\right.$, $I_{T}=100 \mathrm{pA}$, scan area $=12.3 \mathrm{~nm} \times 7.0 \mathrm{~nm}$ ). (b) The $\mathrm{STM}$ image, superimposed by the atomic model of the topmost layer (Si: large green balls; O: small red balls). The bar below indicates the crystalline and the vitreous areas. The black arrow shows the direction of the interface analysis.

model of the film's topmost layer is superimposed onto the STM image in Fig. 1(b) (Si: large green balls; O: small red balls-for coordinates, see Supplemental Material [24], Table S1). This atomic model served as a starting point for further analysis of the crystalline-vitreous interface.

A closer look at the model shows that the left part of the image consists of a regular and periodic arrangement of atoms. However, the right third of the image lacks periodicity and symmetry and is therefore vitreous. Later, we will define a quantity that gives a measure of the order in a particular film region.

It is important to note that we did not observe any electronic signature from the crystalline-vitreous interface in the STM under the given tunneling conditions [see, e.g., Fig. 1(a)]. For the antiphase domain boundaries in ultrathin alumina on $\mathrm{NiAl}(110)$, pronounced electronic features were observed, which were attributed to defect-induced states of the nonstoichiometric structure at the interface [29]. We therefore conclude an absence of such defect states at the crystalline-vitreous boundary of the thin silica film. There are also no under- or overcoordinated $\mathrm{Si}$ atoms, as all $\mathrm{Si}$ atoms have exactly three $\mathrm{Si} \mathrm{NNs}$ (four NNs if one takes into account the Si atom of the first silica layer sitting underneath every $\mathrm{Si}$ atom of the second layer that is imaged). Thus, the stoichiometry is conserved at the crystalline-vitreous transition. These considerations show that the vitreous patch is smoothly connected to the crystalline one.

To evaluate the transition between both regions, it is interesting to look at the change of quantities that characterize the film's structure as we move from the crystalline to the vitreous area. For this Letter, we investigated the NN distances, $\mathrm{NN}$ orientations, and ring statistics. It would be desirable to evaluate these quantities perpendicular to the interface. However, it is difficult to define the exact boundary line, because it is impossible to say whether a sixfold ring belongs to the crystalline or the vitreous region, as they appear in both phases. Therefore, we chose to evaluate the interface structure by approaching it from a direction that is perpendicular to one crystalline axis [see the black arrow below Fig. 1(b)]. The image was cut into vertical slices. Subsequently, the characteristic quantities were computed for every slice separately.

First of all, we evaluated the Si-Si NN distances to tell how the atomic separations change at the interface between the crystalline and the vitreous phase. The distances of all Si-Si NN pairs were computed and are displayed in Fig. 2(a). Differently colored bars represent the magnitude of the Si-Si NN distance [see the scale bar in Fig. 2(a)].

(a)

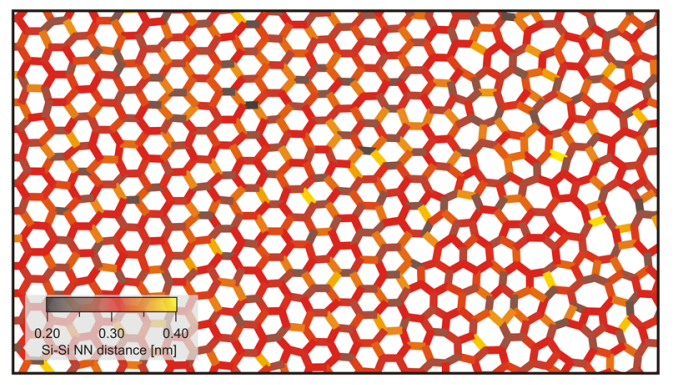

(b)

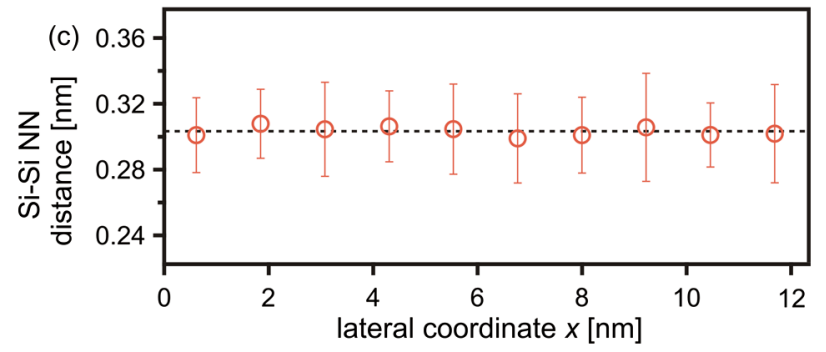

FIG. 2 (color online). Evaluation of the Si-Si distances. (a) The $\mathrm{Si}-\mathrm{Si} \mathrm{NN}$ distances are visualized by colored bars. The color scale represents the distance between two Si atoms (see the scale bar). (b) The position of ten vertical, $1.23 \mathrm{~nm}$ wide, and $6.95 \mathrm{~nm}$ high slices. (c) The average $\mathrm{Si}-\mathrm{Si} \mathrm{NN}$ distances for every slice are plotted vs the lateral coordinate of the respective slice center. Error bars represent the standard deviation of a Gauss fit to the distance distribution. The dashed line specifies the average value for the whole image $(0.303 \mathrm{~nm} \pm 0.025 \mathrm{~nm})$. 
Figure 2(a) shows a homogeneous distribution of Si-Si NN distances throughout the whole image including the crystalline, the vitreous, and the interface region. For a better quantification of the Si-Si NN distance evolution, we divided the image into ten vertical, $1.23 \mathrm{~nm}$ wide slices and computed the distance distribution for every slice. Each distribution was fitted by a Gaussian yielding peak values and standard deviations. Figure 2(c) shows a plot of these two quantities vs the lateral coordinate of the respective slice $(x)$. Although the slices' mean values exhibit a slight variation of $0.003 \mathrm{~nm}$ around the average for the whole image $(0.303 \mathrm{~nm} \pm 0.025 \mathrm{~nm}$, dashed line $)$, the deviation is not significant. Therefore, we conclude that the mean Si$\mathrm{Si} \mathrm{NN}$ distance stays constant as we go from a crystalline to a vitreous state. The NN distance is also not affected at the crystalline-vitreous transition region. This finding is consistent with experimental results on bulk silica materials: the average $\mathrm{Si}-\mathrm{Si} \mathrm{NN}$ distances in vitreous silica [30] and $\alpha$ quartz [31,32] are equal within the root mean square variation of the glass $(0.3077 \mathrm{~nm} \pm 0.0111 \mathrm{~nm}$ and $0.3059 \mathrm{~nm}$, respectively).

Another way of looking at the crystalline-vitreous interface is by exploring characteristic angles between the atoms of the silica film. A quantity that reflects the order of a certain region is the Si-Si NN directed distance orientation (DDO), i.e., the slope of the connection line between two Si NNs with respect to the image abscissa. Figure 3(a) visualizes the different directions present in the film using colored bars. Si-Si NN DDO values range from $-90^{\circ}$ to $+90^{\circ}$ [see also the scale bar in Fig. 3(a)]. Clearly, the DDOs are different for the crystalline and the vitreous phase. This difference is demonstrated in Fig. 3(c), where the DDO values are plotted vs $x$. In the crystalline region (left part of image), DDOs assume three values: $-60^{\circ}, 0^{\circ}$, and $+60^{\circ}$. These three directions reflect the threefold symmetry of the crystalline structure. However, in the vitreous region (right part of image) DDO values scatter randomly from $-90^{\circ}$ to $+90^{\circ}$. This shows that, in this area, the atoms are not collectively aligned. In addition, the representation in Fig. 3(c) gives a clue about the location of the interface region, namely around $x=8 \mathrm{~nm}$.

Surprisingly, in the crystalline region of Figure 3(a), the $\mathrm{NN}$ orientations are not perfectly aligned to each other, but they rather seem to scatter around mean values. This is also visible in Fig. 3(c), where the crystalline peaks $(x<8 \mathrm{~nm})$ exhibit a certain width of roughly $20^{\circ}$ to $30^{\circ}$. In addition, rowlike features of parallel DDOs can be identified in Fig. 3(a) (see, e.g., rows marked by black arrows). We exclude a drift-related effect, because the directions in these rows alternate from row to row. One possible explanation might be that the vitreous area induces stress into the crystalline phase, which is subsequently released via these rows.

The final stage of the crystalline-vitreous interface evaluation is to examine larger objects than $\mathrm{NN}$ distances:

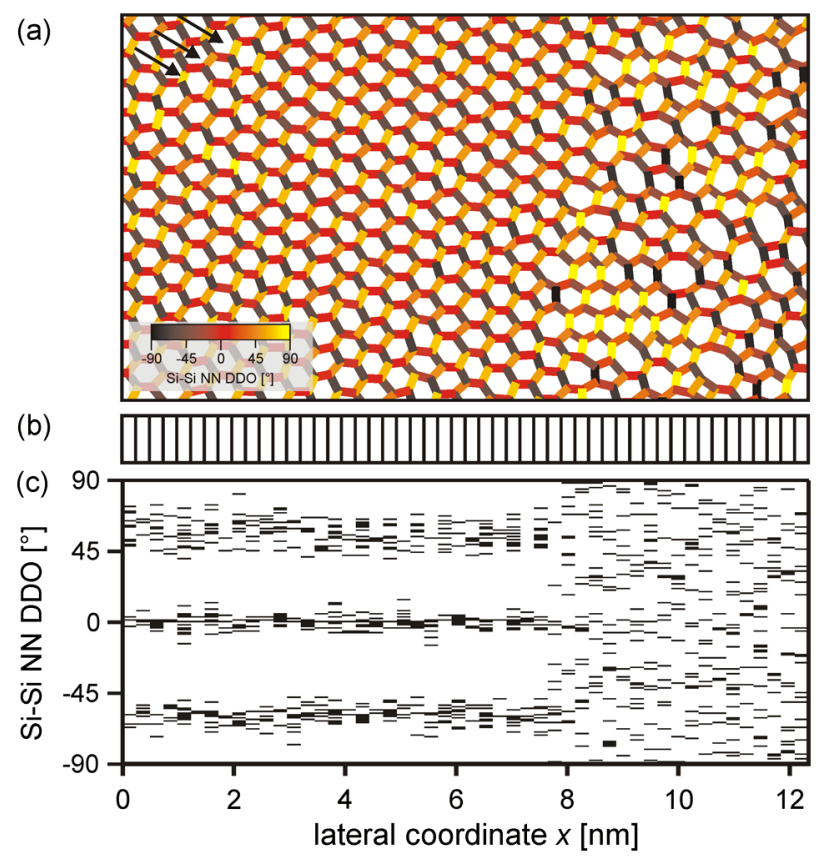

FIG. 3 (color online). Evaluation of Si-Si NN DDOs. (a) The orientation of the directed $\mathrm{Si}-\mathrm{Si} \mathrm{NN}$ distances is displayed using different colors. The color scale indicates the orientation of $\mathrm{Si}-\mathrm{Si}$ line segments with respect to the abscissa of the image plot (see the scale bar). Arrows show examples of rows in the crystalline phase, where the DDOs slightly alternate. (b) The position of 50 vertical, $0.25 \mathrm{~nm}$ wide, and $6.95 \mathrm{~nm}$ high slices. (c) DDO values for every slice plotted vs the slice's lateral coordinate.

the silica rings. By counting the number of $\mathrm{Si}$ atoms involved in every ring, we obtained a $2 \mathrm{D}$ distribution of ring sizes. Furthermore, to gain another quantity that characterizes the ring size, we calculated the areas of the polygons that are defined by the atoms of every ring.

In Fig. 4(a), all rings in the model were colored corresponding to their size. To see even small variations in the size distribution, the color scale was set proportional to the rings' polygonal area (see the scale bar). It becomes clear that the imaged area is not at all homogeneous. Whereas the film consists of only six-membered rings on the left side, rings of various size dominate the right third of the figure. Notably, even the six-membered rings of the crystalline area exhibit a variation in their polygon area. This stems from small deviations of $\mathrm{NN}$ distances and orientations leading to deformations of the rings.

To evaluate how the ring statistics evolve from the crystalline to the vitreous region, we divided the image into 30 vertical slices (each $0.41 \mathrm{~nm}$ wide). Afterwards, we computed ring size distributions for every slice, including ring fractions. Finally, we obtained the 3D data set

$$
N(x, s) \text {, }
$$

where $N$ is the amount of rings per slice, $x$ the lateral coordinate of the respective slice, and $s$ the amount of $\mathrm{Si}$ atoms per ring, i.e., the ring size. In Fig. 4(c), this 3D data 


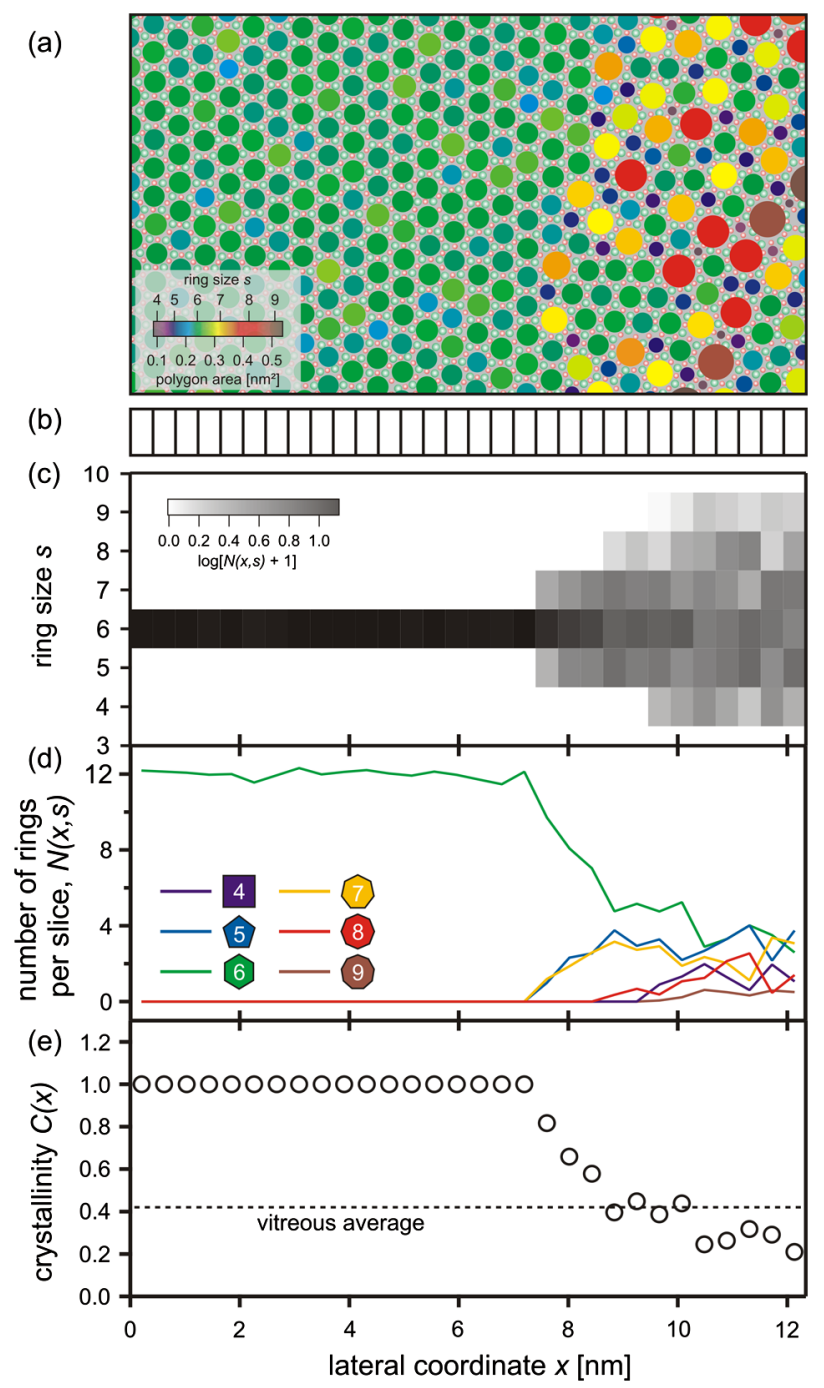

FIG. 4 (color online). Analysis of the rings at the crystallinevitreous interface of the silica film. (a) Visualization of the different ring sizes. Polygon areas range from 0.1 to $0.5 \mathrm{~nm}^{2}$ and ring sizes from four to nine $\mathrm{Si}$ atoms per ring (see the scale bar). (b) The position of 30 vertical, $0.41 \mathrm{~nm}$ wide, and $6.95 \mathrm{~nm}$ high slices. (c) The ring size distribution plotted for every slice. A logarithmic gray scale is used. (d) The number of rings per slice for every ring size plotted vs the lateral coordinate. (e) The crystallinity of every slice plotted vs lateral coordinate. The dashed horizontal line represents the overall crystallinity value of a vitreous region ( $C=0.42$; see Supplemental Material [24] for a detailed analysis).

set is plotted as a 2D graph. To obtain good contrast, the gray scale of the boxes represents the logarithm of the number of rings per slice, $\log [N(x, s)+1]$ (the +1 is added to avoid divergence at zero). Figure 4(d) is a slightly different way to visualize the data. In this graph, $N(x, s)$ is plotted as a curve for every ring size separately [curve colors correspond to the color scale in Fig. 4(a)]. The representations of Figs. 4(c) and 4(d) show an interesting feature of the crystalline-vitreous interface: the first rings to appear close to the crystalline area other than sixfold rings are five- and seven-membered rings. This is in line with density functional theory calculations where a simple model of an amorphous film has been produced out of a crystalline phase by rotation of one of the $\left(\mathrm{SiO}_{2}\right)_{4}$ units [22], leading to the transformation of four six-membered rings into two five- and two seven-membered rings. The prominent appearance of such ring structures has also been observed in other oxide film systems [33]. At increasing lateral coordinate, also four- and eightfold rings are found. The last ring to occur is the ninefold ring, which represents the largest deviation from the presumably energetically most favorable six-membered ring [22].

To better quantify how the film transforms from a crystalline to a vitreous state, we define the crystallinity of a slice

$$
C(x)=\frac{N(x, 6)}{\sum_{s=4}^{9} N(x, s)} .
$$

This quantity is plotted in Fig. 4(e). On the left side of the image $(x \leq 7.2 \mathrm{~nm})$, where the film consists of sixfold rings only, $C(x)=1$. As other ring types start to appear $(x>7.2 \mathrm{~nm}), C(x)$ drops gradually to a value below 1 . The dashed line in Fig. 4(e) marks the reference crystallinity value for a large vitreous area $(C=0.42$; see Supplemental Material [24] for a detailed analysis).

The $C(x)$ plot shows that the thickness of the transition region from crystalline $[C(x)=1]$ to vitreous $[C(x)=$ $0.42]$ is approximately $1.6 \mathrm{~nm}$. For the crystal-glass transitions of other 3D tetrahedral networks, interface widths of 0.3 to $1.4 \mathrm{~nm}$ were obtained theoretically $[4,6,7,14]$ and experimentally $[3,10,20]$. However, it is not straightforward to compare these values to our measurements because of the different interface system (silica-silica) and the different dimensionality of the boundary (2D vs 1D). Furthermore, the thickness of the 1D interface in the silica film might be influenced by small crystalline patches enclosed in the vitreous structure that enhance the crystallinity of the transition region [see, e.g., the bottom right part of Fig. 4(a)]. As a recent Letter shows, local crystallites might also be present in bulk glasses [34].

In this Letter, we studied the atomic structure of the topological transition from a crystalline to a vitreous phase in the thin silica film on $\mathrm{Ru}(0001)$. A smooth interface without under- or overcoordinated $\mathrm{Si}$ atoms was observed. The Si-Si distances appeared to remain constant in the crystalline, the vitreous, and the interfacial region. However, the orientation of the $\mathrm{Si}-\mathrm{Si}$ directed distances showed a substantial change at the interface. Whereas in the crystalline area the angular distribution exhibited three discrete peaks representing the crystalline axes, in the vitreous part the orientations were randomly distributed in all directions. Finally, ring statistics were computed for narrow image slices. This evaluation revealed that five- and sevenfold rings lie closer to the crystalline phase than 
four-, eight-, and nine-membered rings. Furthermore, a thickness of the transition region of about $1.6 \mathrm{~nm}$ was obtained.

We thank Christin Büchner, Stefanie Stuckenholz, and Gero Thielsch for experimental support and fruitful discussions.

*heyde@fhi-berlin.mpg.de

[1] F. Spaepen, Acta Metall. 26, 1167 (1978).

[2] N. Borgardt, B. Plikat, M. Seibt, and W. Schröter, Ultramicroscopy 90, 241 (2002).

[3] N. I. Borgardt, B. Plikat, W. Schröter, M. Seibt, and T. Wagner, Phys. Rev. B 70, 195307 (2004).

[4] N. Bernstein, M. J. Aziz, and E. Kaxiras, Phys. Rev. B 58, 4579 (1998).

[5] L. Houben, M. Luysberg, P. Hapke, R. Carius, F. Finger, and H. Wagner, Philos. Mag. A 77, 1447 (1998).

[6] Y. Tu, J. Tersoff, G. Grinstein, and D. Vanderbilt, Phys. Rev. Lett. 81, 4899 (1998).

[7] C. R. S. da Silva and A. Fazzio, Phys. Rev. B 64, 075301 (2001).

[8] S. Blonski and S. H. Garofalini, J. Am. Ceram. Soc. 80, 1997 (1997).

[9] W. Walkosz, R. F. Klie, S. Öğüt, B. Mikijelj, S.J. Pennycook, S. T. Pantelides, and J. C. Idrobo, Phys. Rev. B 82, 081412 (2010).

[10] D. E. Aspnes and J. B. Theeten, J. Electrochem. Soc. 127, 1359 (1980).

[11] I. Ohdomari, T. Mihara, and K. Kai, J. Appl. Phys. 59, 2798 (1986).

[12] A. Ourmazd, D. W. Taylor, J. A. Rentschler, and J. Bevk, Phys. Rev. Lett. 59, 213 (1987).

[13] F. J. Himpsel, F. R. McFeely, A. Taleb-Ibrahimi, J. A. Yarmoff, and G. Hollinger, Phys. Rev. B 38, 6084 (1988).

[14] A. Pasquarello, M. S. Hybertsen, and R. Car, Appl. Phys. Lett. 68, 625 (1996).

[15] D.-A. Luh, T. Miller, and T.-C. Chiang, Phys. Rev. Lett. 79, 3014 (1997).

[16] A. Pasquarello, M. S. Hybertsen, and R. Car, Nature (London) 396, 58 (1998).

[17] Y. Tu and J. Tersoff, Phys. Rev. Lett. 84, 4393 (2000).

[18] N. Ikarashi, K. Watanabe, and Y. Miyamoto, Phys. Rev. B 62, 15989 (2000).
[19] R. Buczko, S. J. Pennycook, and S. T. Pantelides, Phys. Rev. Lett. 84, 943 (2000).

[20] M.C. Cheynet and T. Epicier, Philos. Mag. 84, 1753 (2004).

[21] N. Nakanishi, Y. Kikuchi, T. Yamazaki, E. Okunishi, K. Watanabe, and I. Hashimoto, Phys. Rev. B 70, 165324 (2004).

[22] L. Lichtenstein, C. Büchner, B. Yang, S. Shaikhutdinov, M. Heyde, M. Sierka, R. Włodarczyk, J. Sauer, and H.-J. Freund, Angew. Chem., Int. Ed. Engl. 51, 404 (2012).

[23] J. F. Shackelford and B. D. Brown, J. Non-Cryst. Solids 44, 379 (1981).

[24] See Supplemental Material at http://link.aps.org/ supplemental/10.1103/PhysRevLett.109.106101 for a similar evaluation of distances, DDOs and the crystallinity of a large vitreous region, the ring size distribution of the vitreous phase, and coordinates.

[25] P. Y. Huang, S. Kurasch, A. Srivastava, V. Skakalova, J. Kotakoski, A. V. Krasheninnikov, R. Hovden, Q. Mao, J. C. Meyer, J. Smet, D. A. Muller, and U. Kaiser, Nano Lett. 12, 1081 (2012).

[26] D. Löffler, J. J. Uhlrich, M. Baron, B. Yang, X. Yu, L. Lichtenstein, L. Heinke, C. Büchner, M. Heyde, S. Shaikhutdinov, H.-J. Freund, R. Włodarczyk, M. Sierka, and J. Sauer, Phys. Rev. Lett. 105, 146104 (2010).

[27] L. Lichtenstein, M. Heyde, and H.-J. Freund, J. Phys. Chem. C (in press).

[28] B. Yang, W.E. Kaden, X. Yu, J. A. Boscoboinik, Y. Martynova, L. Lichtenstein, M. Heyde, M. Sterrer, R. Włodarczyk, M. Sierka, J. Sauer, S. Shaikhutdinov, and H.-J. Freund, Phys. Chem. Chem. Phys. 14, 11344 (2012).

[29] N. Nilius, M. Kulawik, H.-P. Rust, and H.-J. Freund, Phys. Rev. B 69, 121401 (2004).

[30] D. I. Grimley, A. C. Wright, and R. N. Sinclair, J. NonCryst. Solids 119, 49 (1990).

[31] L. Levien, C. T. Prewitt, and D. J. Weidner, Am. Mineral. 65, 920 (1980).

[32] M. G. Tucker, D. A. Keen, and M. T. Dove, Mineral Mag. 65, 489 (2001).

[33] F. Yang, Y. Choi, P. Liu, D. Stacchiola, J. Hrbek, and J. A. Rodriguez, J. Am. Chem. Soc. 133, 11474 (2011).

[34] J. Hwang, Z.H. Melgarejo, Y.E. Kalay, I. Kalay, M. J. Kramer, D. S. Stone, and P. M. Voyles, Phys. Rev. Lett. 108, 195505 (2012). 